\title{
T Helper 17 Cell
}

National Cancer Institute

\section{Source}

National Cancer Institute. T Helper 17 Cell. NCI Thesaurus. Code C113815.

A subset of helper T-lymphocytes which synthesize and secrete the interleukins (IL), IL17A, -21 and -22 . These cytokines mediate inflammation by increasing chemokine production, which leads to the recruitment of both monocytes and neutrophils to sites of infection or inflammation. Th17 cells modulate both host immunity against extracellular bacteria and fungi and inflammation. Overexpression of these cells is associated with tissue injury caused by autoimmune diseases. 